\title{
INVERSIÓN SOCIAL: Enfoque para clasificar los proyectos
}

\begin{abstract}
RESUMEN
El presente artículo brinda un enfoque conceptual de los proyectos sociales y los proyectos de inversión social, diferenciando sus enfoques, conceptos y metodologías para una mejor comprensión y aplicación en el campo del desarrollo social.
\end{abstract}

Palabras clave: Inversión social, proyectos sociales, proyectos de inversión social.

SOCIAL INVESTMENT: APPROACH TO RANK PROJECTS

\section{ABSTRACT}

This article provides a conceptual approach to social projects and social investment projects, differentiating their approaches, concepts and methodologies for better understanding and application in the field of social development.

Keywords: Social investment, social projects, social investment projects.

\section{INTRODUCCIÓN}

La inversión pública en nuestro país en los últimos años ha mostrado un incremento considerable debido principalmente a la situación económica por la que atraviesa el país, por ello se han desarrollado un conjunto de proyectos como infraestructura educativa y de salud, fortalecimiento de capacidades, carreteras, saneamiento, etc. La diversidad de intervenciones realizadas por los organismos públicos ha generado denominaciones a los proyectos, a los que es necesario clasificarlos para utilizar las herramientas adecuadas en cada una de ellas.

Por ello, es imprescindible ordenar conceptualmente las intervenciones catalogadas como proyectos en el sector público y establecer las metodologías adecuadas en su elaboración, el cual es el objetivo del presente artículo.

\section{INVERSIÓN SOCIAL}

La inversión en nuestro país se ha destinado a un conjunto de proyectos que por su naturaleza se han desarrollado en distintos sectores para mejorar directa o indirectamente las condiciones de vida de la población, a ella se le denomina inversión social.

\section{Índice de Desarrollo Social}

El Ministerio de la Mujer y Desarrollo Social publica en el año 2010 el documento que sustenta el índice de desarrollo social. Es un indicador que señala el nivel de desarrollo de las capacidades de interacción, coordinación, organización y acción social del país, alcanzadas a partir de la implementación de Planes, Programas, Estrategias y acciones que buscan ofrecer a la población oportunidades de mejoramiento de su calidad de vida.

Las dimensiones que constituyen la base para la selección de los respectivos indicadores que conforman el índice del nivel de desarrollo social del país son:

Bienestar e ingreso: Participar en la actividad económica y gozar de condiciones adecuadas de inserción laboral que permitan ingresos suficientes para lograr un nivel de vida digno.

Ingeniero Industrial UNMSM, Profesor en la Facultad de Ingeniería Industrial, Departamento Académico de Producción y Gestión Industrial de la UNMSM. E-mail: cieandia@hotmail.com 
Salud y Nutrición: Orientado a gozar de una vida sana y saludable, lo que implica contar y tener acceso a redes formales de servicios de salud y seguridad social así como a una nutrición apropiada, que garanticen una adecuada calidad de vida de la población.

Educación: Relacionado con la disponibilidad y el adecuado acceso de la población a los servicios de educación y capacitación que favorezcan el desarrollo del capital humano.

Participación social: Reflejado en el desarrollo de procesos cívicos nacionales y locales, para que se desarrolle en la población el sentido de pertenencia y de cohesión social, y con ello, el sentimiento de participación activa y responsable que implica el deber y el derecho de los ciudadanos a participar en el mismo.

Servicios básicos: Son indicadores que procuran la calidad de la salud, ya que involucran buenas prácticas de higiene y limpieza. El acceso a agua potable, desagüe, electricidad y morar en una vivienda confortable en relación al nivel socioeconómico alcanzado.

Seguridad: Relacionado al libre desenvolvimiento y desarrollo de actividades públicas y privadas sin temor de ser víctima de delito.

\section{Mapa del Índice de Desarrollo Social}

El Mapa del Desarrollo Social representa un aporte importante porque constituye una propuesta de clasificación de los departamentos en virtud de sus características específicas en cuanto a las dimensiones consideradas en el cálculo del IDS. Ello da lugar a una tipología de departamentos que las clasifica en cinco niveles: Nivel Alto, Medio Alto, Medio, Bajo y Muy Bajo de desarrollo social alcanzado.

Tabla N. ${ }^{\circ} 1$. Índice de Desarrollo Social por departamentos.

\begin{tabular}{|l|c|l|}
\hline Departamentos & IDS & Clasificación \\
\hline Tacna & 0.702 & Alto \\
\hline Lima 1/ & 0.641 & Alto \\
\hline Ica & 0.613 & Alto \\
\hline Moquegua & 0.612 & Alto \\
\hline Arequipa & 0.591 & Medio Alto \\
\hline Junín & 0.588 & Medio Alto \\
\hline Madre de Dios & 0.568 & Medio Alto \\
\hline La Libertad & 0.520 & Medio Alto \\
\hline
\end{tabular}

\begin{tabular}{|l|l|l|}
\hline Pasco & 0.516 & Medio \\
\hline Tumbes & 0.513 & Medio \\
\hline Ancash & 0.497 & Medio \\
\hline Piura & 0.492 & Medio \\
\hline Cusco & 0.472 & Medio \\
\hline Lambayeque & 0.471 & Medio \\
\hline Amazonas & 0.467 & Bajo \\
\hline San Martín & 0.467 & Bajo \\
\hline Puno & 0.460 & Bajo \\
\hline Loreto & 0.438 & Bajo \\
\hline Ucayali & 0.431 & Bajo \\
\hline Apurímac & 0.424 & Muy Bajo \\
\hline Huánuco & 0.419 & Muy Bajo \\
\hline Cajamarca & 0.411 & Muy Bajo \\
\hline Huancavelica & 0.411 & Muy Bajo \\
\hline Ayacucho & 0.390 & Muy Bajo \\
\hline 1/ Incluye la Provincia Constitucional del Callao. \\
\hline
\end{tabular}

Fuente: MINDES-DGPDS.

\section{CLASIFICACIÓN DE PROYECTOS}

Desde una perspectiva de Desarrollo Social, el proyecto es un conjunto de acciones dirigidas a producir cambios, a transformar la realidad, la situación o condición de un grupo social determinado.

Para lograr el desarrollo social en una región es necesario que se realicen intervenciones en forma directa (proyectos de inversión y proyectos sociales) e indirecta (proyectos complementarios).

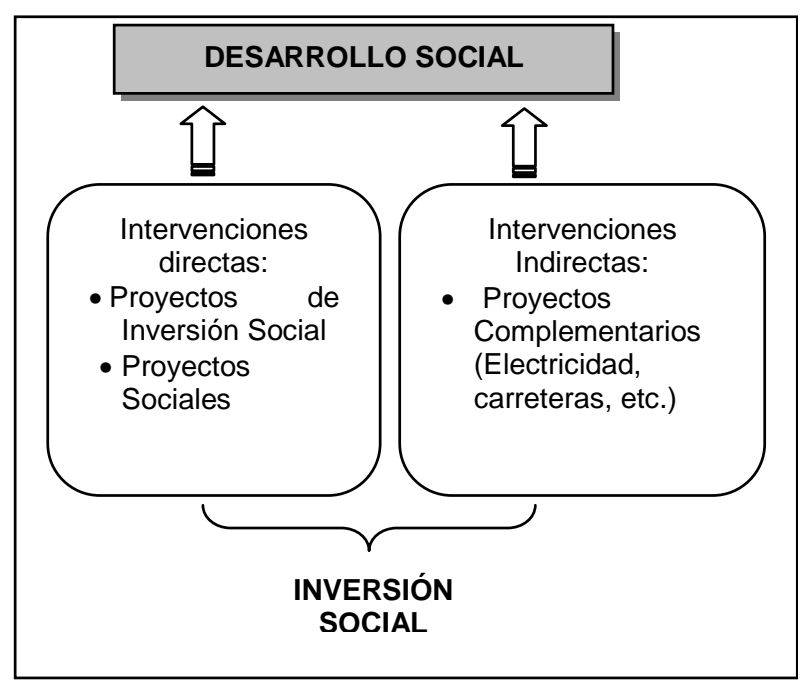

Figura N. ${ }^{\circ}$. El Desarrollo Social 


\section{a. Proyectos de Inversión Social}

Son proyectos cuyo beneficio está centrado en forma directa en las personas, en su bienestar y en el mejoramiento de sus condiciones de vida. Además, cumplen con el requisito de un proyecto de inversión (ciclo de vida: preinversión, inversión y postinversión). Por ejemplo, proyecto de ampliación de servicios de un Centro de Emergencia Mujer, cuyos componentes son infraestructura, equipamiento, capacitación, sensibilización, etc.

Los proyectos de inversión social se inician con la elaboración de estudios ya sean a nivel de perfil, prefactibilidad o factibilidad; luego se ejecutan, es decir se implementan para brindar o mejorar un servicio permanente (etapa de postinversión), en donde se generan costos de operación y mantenimiento.
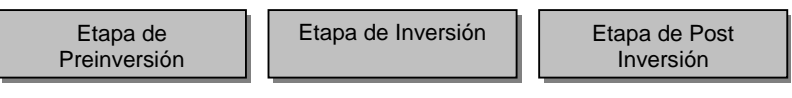

Figura. N. ${ }^{\circ}$ 2. Ciclo de vida de un Proyecto de Inversión.

\section{b. Proyectos Sociales}

Son proyectos cuyo beneficio está centrado en forma directa en las personas, su bienestar y el mejoramiento de sus condiciones de vida, aunque no se enmarcan como proyectos de inversión (no cumplen el ciclo de vida), tienen el mismo objetivo de la perspectiva de desarrollo social. Es decir, es un conjunto de acciones articuladas de instituciones nacionales o internacionales destinadas a producir cambios en una determinada realidad que involucra y afecta a un grupo social determinado.

Debido a su naturaleza, los proyectos sociales buscan resolver, en forma directa, los problemas de las personas como la exclusión, discriminación, maltrato, etc. en el medio donde se desenvuelven. Sus acciones son principalmente: sensibilización, talleres, soporte emocional, difusión, etc.; las que no cumplen con las características de un proyecto de inversión, específicamente en el ciclo de vida del proyecto las etapas de inversión y post inversión se presentan en forma simultánea. Es decir, en los proyectos sociales se tiene un ciclo de vida distinto, sus etapas son: diseño e implementación.

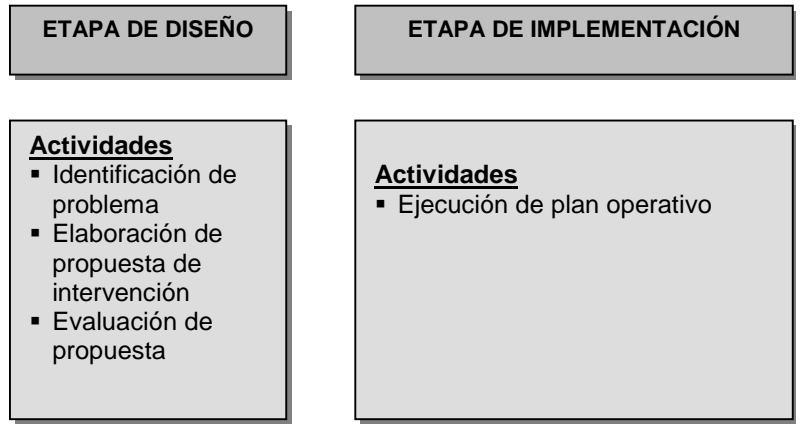

Figura N. ${ }^{\circ}$ 3. Ciclo de vida de un Proyecto Social.

\section{Etapa de Diseño}

Es aquella donde se sustenta la problemática a resolver, se define sus características y la estrategia de intervención para lograr su objetivo propuesto, a través de un conjunto de instrumentos y metodologías propias. Por su naturaleza, no requiere de estudios diferenciados en cuanto a su profundidad de análisis, además su presupuesto combina gastos en bienes, servicios y pagos de personal, entre otros, necesarios para la implementación (recordar que en los proyectos de inversión social no se puede acumular gastos de inversión con gastos de la etapa de postinversión).

\section{Etapa de Implementación}

Una vez obtenido los recursos, se inicia la implementación, es decir, el desarrollo de todas las actividades propuestas durante el tiempo de duración del proyecto (frecuentemente los proyectos sociales tienen menor tiempo de duración que los de inversión social), es aquí donde se presentan los cambios a corto y mediano plazo. En los proyectos sociales, la etapa de implementación combina la ejecución y la postinversión del ciclo de los proyectos de inversión social.

En esta etapa se realiza la ejecución de los recursos para obtener los bienes y servicios necesarios para la implementación del proyecto, como: elaboración de materiales, adquisición de equipos, etc. Además se realiza la intervención, es decir, desarrollo de las actividades en el medio social (capacitación, asistencia técnica, talleres, etc.).

En la etapa de implementación se desarrollan los planes operativos, que son instrumentos de gestión que permiten implementar el proyecto en forma coherente, concreta y ordenada, su planificación se estima en periodos anuales. 


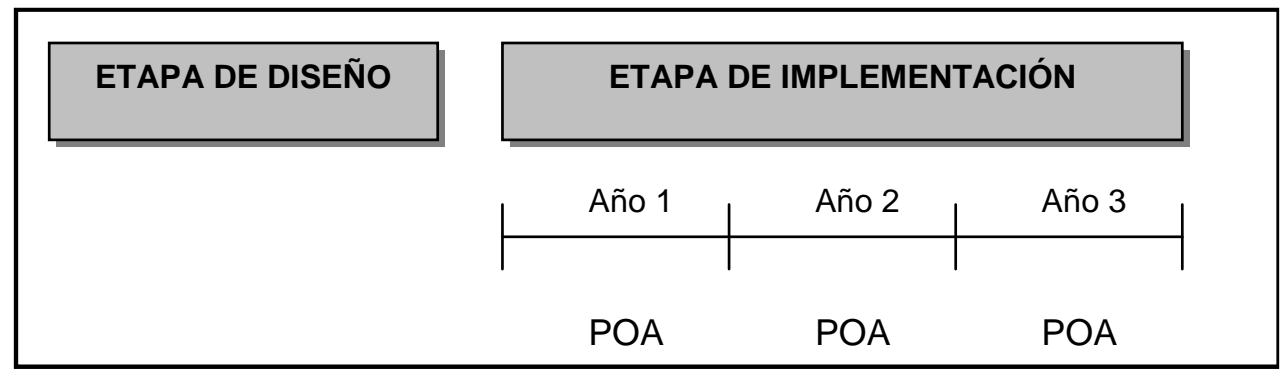

Figura N. ${ }^{\circ}$ 4. El Plan Operativo Anual.

\section{LOS RECURSOS EN LOS PROYECTOS}

Una vez identificado el problema y sus características es necesario determinar su magnitud, es decir, cuantificar el nivel de intervención en cuanto a cobertura, para ello se debe estimar a quienes va ser dirigido el proyecto y la cantidad de bienes o servicios que se va entregar (materiales de capacitación, número de talleres, cursos de capacitación, etc.). Dicha cuantificación se debe realizar en los proyectos de inversión social como en los proyectos sociales, ya que ambos requieren dicha información para cuantificar los recursos necesarios que se van a destinar en el proyecto. En el primer caso, se denomina la inversión del proyecto, para el segundo caso se denomina el costo total del proyecto.

Los recursos necesarios en los Proyectos de Inversión Social

\section{EL CICLO DE VIDA, MARCO LÓGICO Y LA EVA- LUACIÓN DE PROYECTOS}

La congruencia de distintos conceptos y metodologías utilizadas en la formulación y la evaluación de proyectos es muy importante para comprender el proceso lógico de intervención y el momento apropiado de su implementación. Dicha conceptualización se presenta en la Figura $\mathrm{N}^{\circ}{ }^{4}$, en el que se relaciona el ciclo de vida, el marco lógico y la evaluación de proyectos.

Para ello, se considera la evaluación al proceso que permite determinar el logro de los objetivos ya sea a nivel de propósito y en algunas ocasiones a nivel de "Fin", según lo establecido en el diseño del proyecto.

Además, el monitoreo se define como el proceso que permite determinar si el uso de los recursos



Figura N. 5. Inversión del Proyecto.

Los recursos necesarios en los Proyectos Sociales



Figura N. $^{\circ}$ 6. Costo del Proyecto. 
para desarrollar las actividades y obtener los componentes se están desarrollando según lo establecido en el plan operativo. El monitoreo se presenta en la etapa de ejecución del proyecto.

La diferenciación entre los proyectos sociales y los proyectos de inversión permite utilizar el enfoque de sustento adecuado, así como el uso de herra- mientas metodológicas pertinentes para: i) demostrar la viabilidad de la propuesta, ii) el empleo correcto de los términos, iii) determinar la inversión y su financiamiento según corresponda. Es importante resaltar que el Sistema Nacional de Inversión Pública solo financia intervenciones catalogadas como proyectos de inversión.

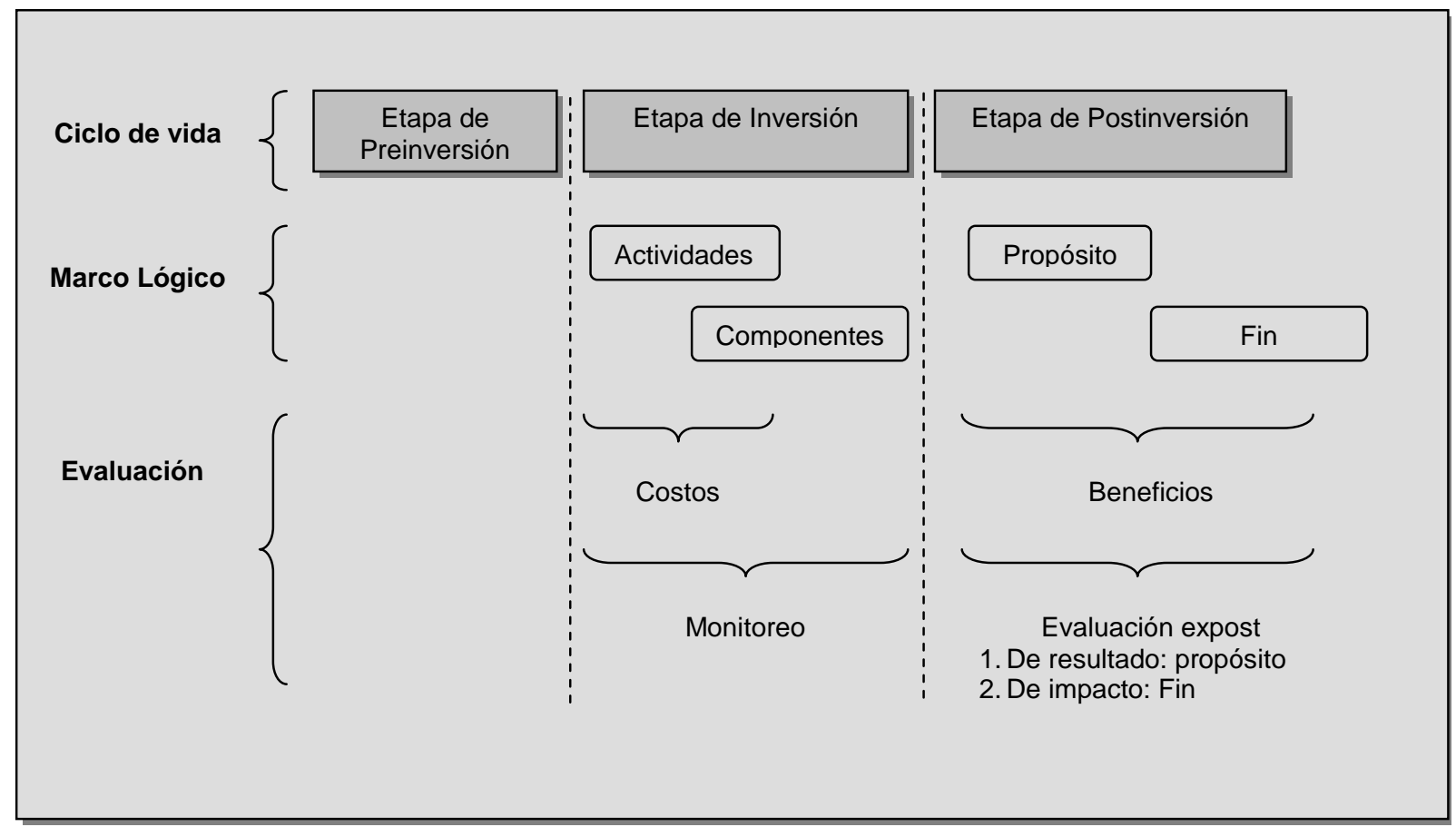

Figura N. 7. Esquema para un Proyecto de Inversión Social.

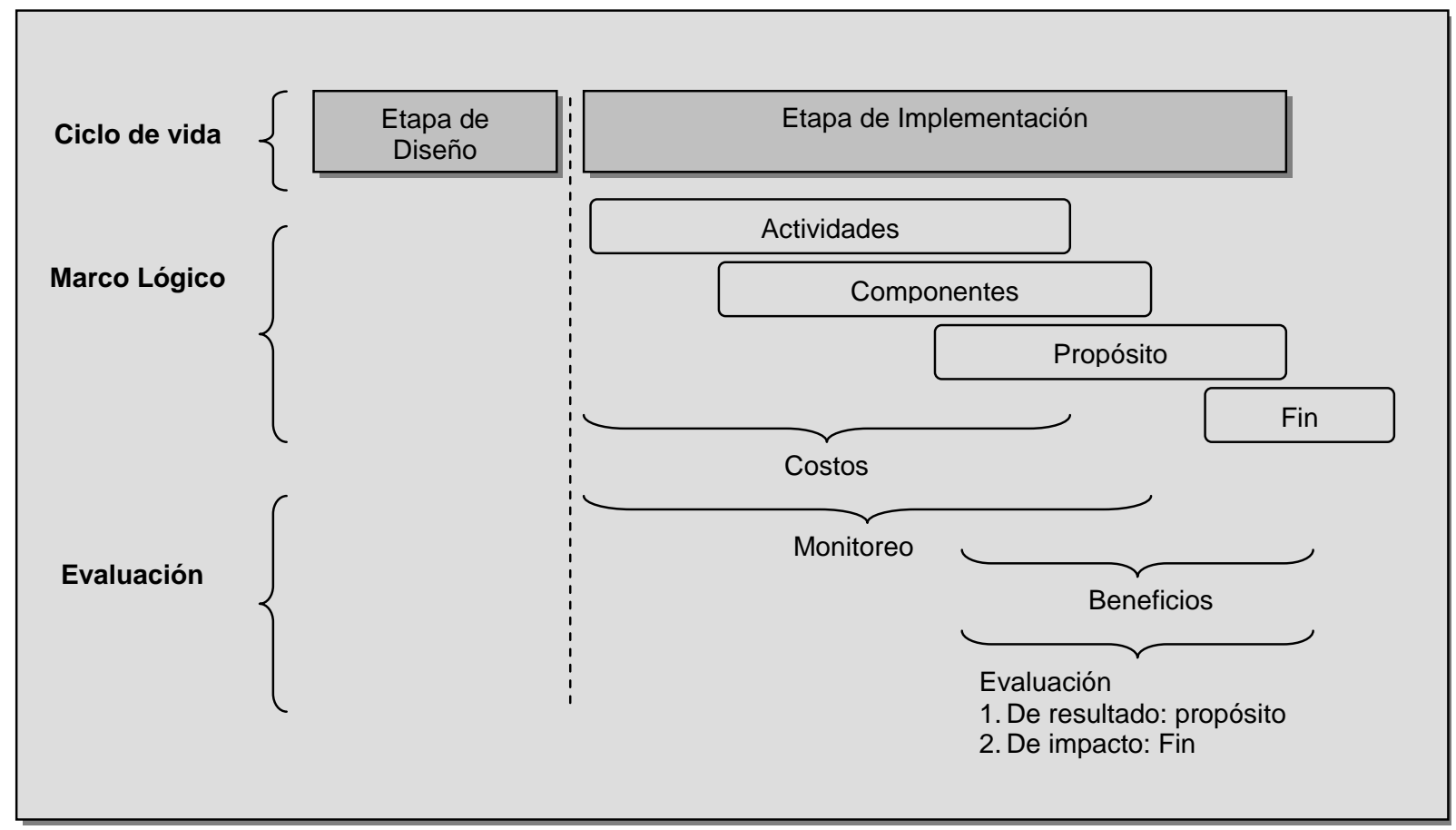

Figura N. ${ }^{\circ}$ 8. Esquema para un Proyecto Social. 


\section{CONCLUSIONES}

1. Los proyectos de inversión social y los proyectos sociales tienen el mismo objetivo pero conceptualmente utilizan distintos enfoques de intervención y metodologías.

2. El ciclo de vida de un proyecto es una herramienta que permite identificar el tipo de proyecto y sus características.

3. Es importante diferenciar los conceptos que se utilizan en los proyectos sociales y de inversión social para evitar la generación de confusiones que lleven a distorsionar dicha disciplina.

\section{REFERENCIAS BIBLIOGRÁFICAS}

[1] Andía Valencia, W. (2010). Gerencia de Proyectos Sociales y de Inversión Social. Primera edición. El Saber Editores. Perú.

[2] Andia Valencia, W. (2009). Proyectos de Inversión. Segunda edición. El Saber Editores. Perú.

[3] Castro, R. y Mokate K. (1994). Evaluación económica de proyectos de inversión, Facultad de Economía, Universidad de los Andes, Colombia.

[4] Cohen, E. y Franco, R. (1992). Evaluación de proyectos sociales. Siglo XXI, México. 